\title{
RECENT TECHNOLOGY FOR TRANSLATION STUDY
}

\author{
Witri Handayani ${ }^{1}$, Yenni Rozimela ${ }^{2}$, Harris Effendi Thahar ${ }^{3}$, Syahrul Ramadhan ${ }^{4}$, \\ Agustina $^{5}$, M. Zaim ${ }^{6}$ \\ ${ }^{1}$ Politeknik Negeri Padang, Padang, Indonesia, $\bowtie \underline{\text { wietripnp@gmail.com }}$ \\ ${ }^{2}$ Universitas Negeri Padang, Padang, Indonesia, $₫$ yennirozi@gmail.com \\ ${ }^{3}$ Universitas Negeri Padang, Padang, Indonesia, $\square$ harrispadang@gmail.com \\ ${ }^{4}$ Universitas Negeri Padang, Padang, Indonesia, $\triangle$ syahrulramadhan.532@gmail.com \\ ${ }^{5}$ Universitas Negeri Padang, Padang, Indonesia, $\square$ tien agustina08@yahoo.com \\ ${ }^{6}$ Universitas Negeri Padang, Padang, Indonesia, $₫ \underline{\text { mzaim unp@yahoo.com }}$
}

\begin{abstract}
This article aimed to offer the recent technology which can be used for translation study. This technology which, surely, mostly provided through the internet access has the purpose to assist the translator especially translation students in finishing their translation works. Basically, the most common assistance comes from these two technology innovations: computer-assisted translation tools (CAT tools) and machine translation (MT). There are many options from this two technologies which can be utilized to ease and smoothen the translation works, such as trados, wordfast and Atril Déjà Vu as the choise for CAT tools and two ranks of commercial and free machine translation as the choice for MT. This two technologies have been developt significantly by the provider. However, human intervention still helds an important influence on their quality improvement. Therefore, human translation as the user of this two recent technologies should know how to wisely used them.
\end{abstract}

Keywords: translation study, CAT tools, machine translation

\section{Introduction}

The work of translation is difficult yet easy at the same time. This perspective may come from different people depend on how they do the work of translation. For instance, two students were doing their translation exercises on the computer with full internet access, the first student directly translated the exercise only by using online and offline dictionary while the second student translated the exercise by maximumly used the internet access; by using search engine and online dictionary simultaneously. As the result, the first student came up with more literal result of the translation than the second student had. Vise versa, other non translation students can master the translation skills autodidactly with the help of technology on the translation tools, eventhough take a long time but it makes the translation job is not a special task left for the special people anymore (Campbell, 2002).

The phenomenon occurs due to two major facts: first, imbalance mastery of translation skill and second language ability. Before doing the translation, the translator should avoid double difficulties in translation by learning the translation skills and learning the second language simultaneously (Benfoughal,2010). To overcome this problem, the translation study has spread up with the purpose to create and train a translator with better translation skills. Many books and articles about translation works also has been released to give better perspective and knowledge for translation students and the one who will to learn about how to translate theoritically.

Second, on this digital era, traditional human translation surely cannot cope the translation need today (O'Reilly, 2005). The utilization of dictionaries and the translators' brain only cannot guarantee that the result of the translation would match the clients' request because they can compare it with another source in the target language which they can find easily on the internet.Beside, the accepted translation result should not sound like a translation. It should be very natural that nobody recognize it as the translation result (Kenny, 1996). To overcome this problem, many assistances, actually, have been provided on the internet to ease and smoothen the work of translation. Some previous researches have mentioned the utilization of technology in translation works, for example Wikipedia and Knol which is used for donating their translation free and Google Translator Toolkit which require the translation to upload their work on 'cloud' (Pym, 2011). Meanwhile, Patience (2016) mention that there are some tools which commonly used as the important computer tools in the workplace of translation, such as electronic dictionaries, glossaries and terminology databases, concordances, online biligual texts, and translation memories. However, it is acknowledged by limited number of people. Therefore, on this article, all 
assistances which can be gotten from recent technology like the internet and some tools for translation work would be discussed.

\section{Discussion}

There are some assistances which provided by the internet and can be download and access easily. Some of them are even free access, yet for better professional usage, it is suggessted to buy the upgrading ones.

There are two major technological innovations: computer-assisted translation tools and machine translation that are driven the technological development in translation (Doherty, 2016).

\section{Computer-Assisted Translation Tools}

Computer-Assisted Translation Tools (CAT Tools) occured as the answer of industrial need for translating their products on the purpose to be successful on international markets. Some software companies of the 1990s and several other technology-related industies searched for the solution of increase productivity in translation and maintain consistency of their linguistic data across a growing number of languages and countries (Esselink, 2000). This CAT Tools provided the first major technological shift and memory in the present-day translation works.

CAT Tools is not a program based of machine translation and have no ready used dictionary (Cocci, 2009). It works by using a translation memory (TM) which function to store a translator's translated text, so that the next text which have similar linguistic composition can reuse this TM in full or in part (Doherty, 2016). For example, having the previous text result from English to Indonesian:

\section{Turn off instant translation}

Matikan terjemahan instan

TM would save the result as the original, so when the similar linguistic composition text occur, such as 'Turn on instant translation' the choice of the result would be underlined or highlighten.

Turn on instanttranslation

Hidupkan terjemahaninstan

TM can just store the first text by selecting 'New TM', but then on the next text the TM can work by showing the stored translation from the first sentence and highlight the lexical matches then provide an option whether to use the choice or not after clicking the 'select TM'.

Some examples of CAT Tools are: Trados, Wordfast, and Atril DéjàVu (Cocci, 2009). Each tools basically over similar service with different specification. Here are some details for each tools.

\section{Trados}

Trados is the most popular CAT tool in present (Cocci, 2009). Before opening the source text, the Translator's Workbench on the trados should be opened and will remain open during the translation process. Next, the tools will automatically segmented the text based on its punctuation marks. However, the segments can be adjusted longer or shorther, depending on the text type and translator preferences.

Trados locates both the source text and target text in the same area of the screen, mark them with different colors and have same type size and typeface. Once the translation has been inserted in the bottom window, the program saves both source and translated segments in the TM. When the same segment occurs in this or future translation, trados can automatically suggest the optional result on the target language. If, during the translation process, a term appears and the translator identify that it is stored in the memory, it will be marked and a Concordance command is entered and function to retrieves all segments in which the term occurs. The example of trados tools is as follows:

Picture 1. Trados 


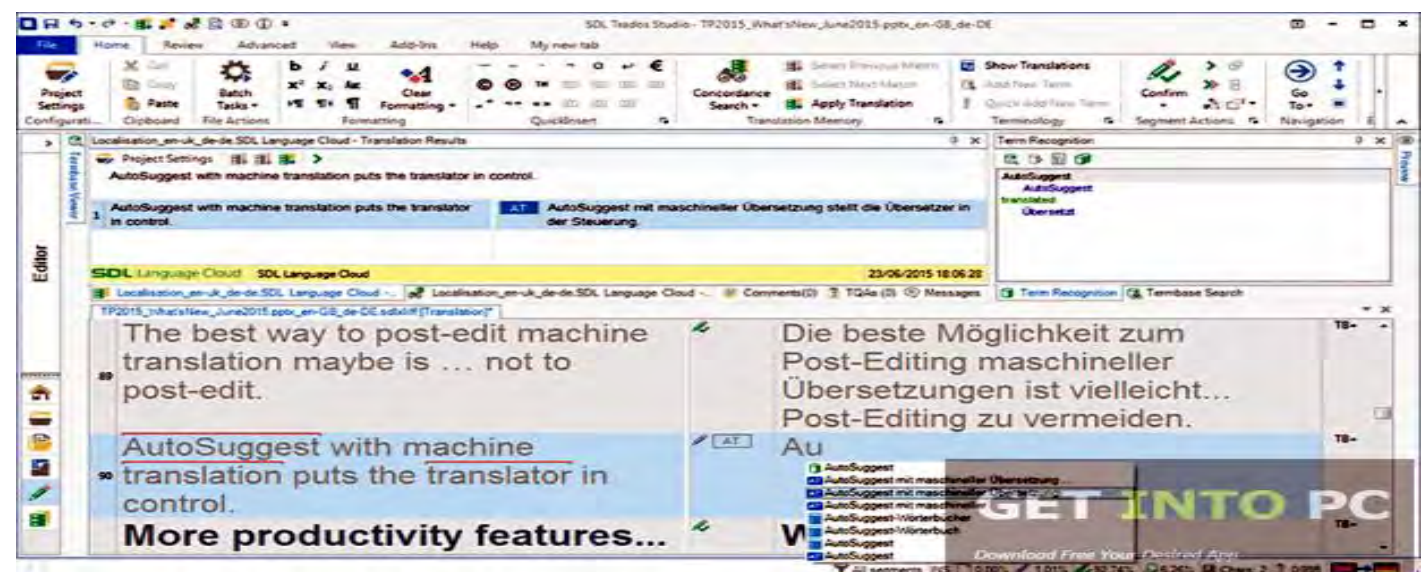

\section{Wordfast}

Wordfast consists of wordfast classic, wordfast pro and wordfast anywhere. Each of them is easy to use with slightly different term inside. Different from trados which offers side-by-side translation works, wordfast divides the segments in different ways, but the basic principles are the same, both of the tools offer the combination of two technologies: segmentation and translation memory (Cocci, 2009).

Wordfast classic is really suitable for the beginner of CAT tools user, especialy students, because it is not actually a program, but a suite of Microsoft Word macros. It provides multiple TMs and glossaries, autosuggest, automatic find-replace, track changes and many other features (Brosure, 2013). However, it handles fewer formats and has limited performance range compared to other CAT tools.

Wordfast Pro is a greatest innovation of the previous versions. The program is already saparated from the Word. The interface is clear, simple and intuitive. The best parts of the innovation are faster analyze and cleanup functions, the retrieval of repeating segments and an expanded range of compatible file format. It is a paid license tools which provides free trial for 30 days and demo mode with full-featured trial version (Brosure, 2017).

Wordfast Anywhere is a tool which frees the translators from their desktop application and allows them to work on their translation projects from anywhere they have a web browser. It also offers each user a private, password-protected workspace on our centralized server. The data is backed up frequently and automatically so you get guarantee that your data will not lose (Brosure, 2013).

This is the example of wordfast classic which is opened by using microsoft word.

Picture 2. Wordfast Classic

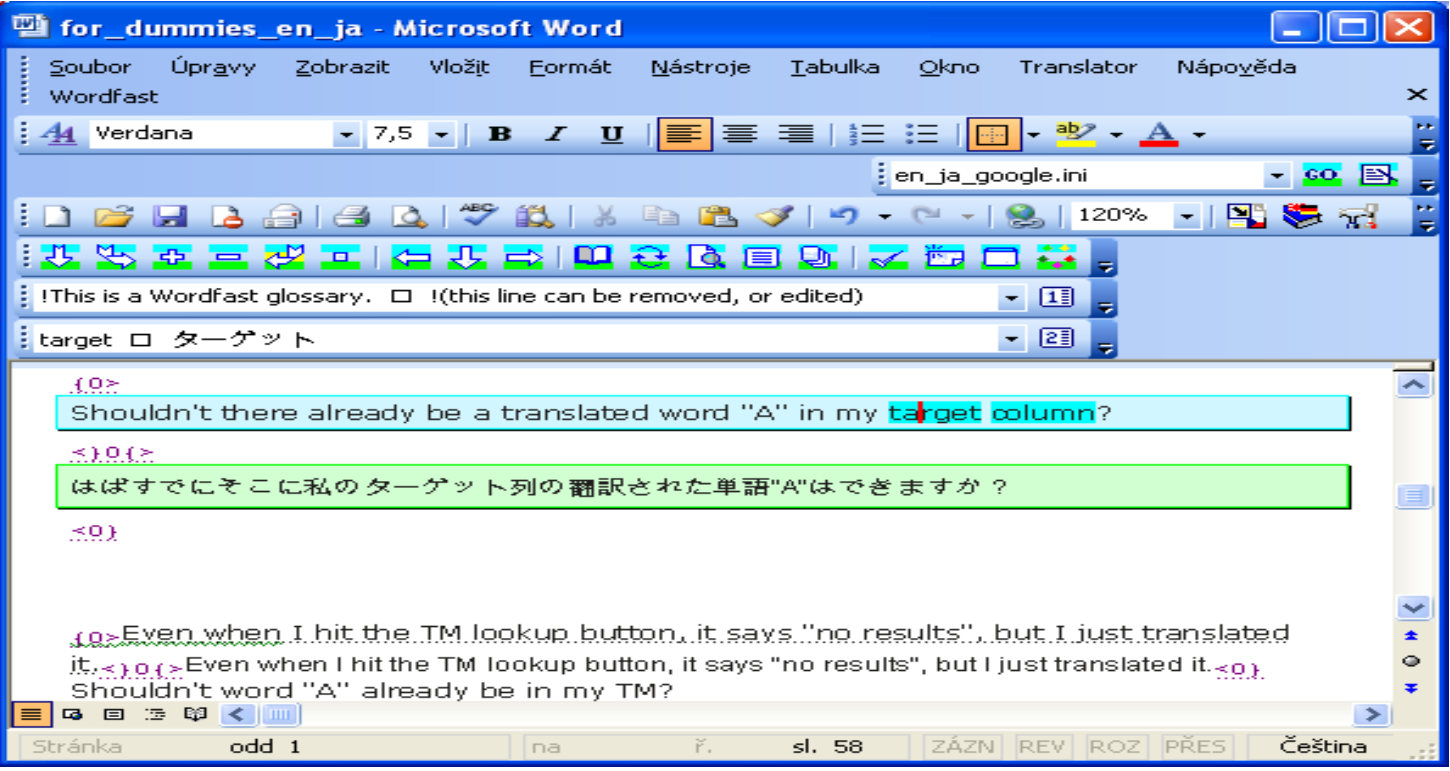

Atril DéjàVu

Atril DéjàVu is a software which has the capability to import hundreds of files in a single operation without the need to change the file structure. A single window can also be used to display multiple files. It also includes 
certain functions, like replace, filter, and viewing lines containing only selected words and expressions which can be applied to all segments pairs (source and result) appearing in a window (Cocci, 2009). In addition, one type of DéjàVu, DéjàVu X2, has an aligment component but minus the visual, drag-and-drop approach to join the relevant segments which could be particularly helpful in language learning (Fernandez-Parra, 2016).

Déjà $\mathrm{Vu}$ has interesting function like EBMT (Example-Based Machine Translation), which few other CAT tools feature. The function is different from what Trados or Wordfast has. Rather than offering the translation result for some similar linguistic compositions for the text, Déjà $V u$ has the capability to 'correcting' the differences which means offering better option. Therefore, it is the ideal solution for those working with very large projects consisting of many files in different formats (e.g., for translating Web pages).

Picture 3. Atril DéjàVu

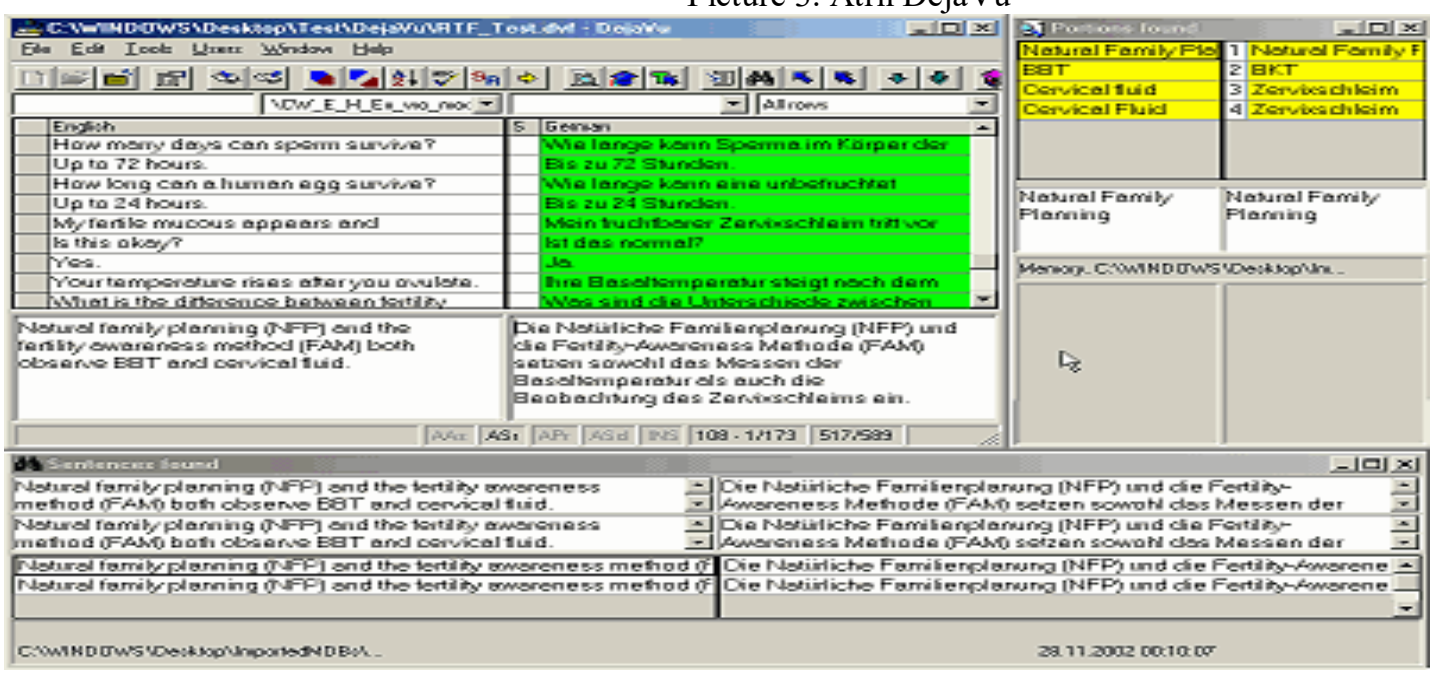

\section{Machine Translation}

The utilization of machine translation in its history had been started in 1930s as mechanical multilingual dictionaries. However, in the year of 1950s, it was known as a limited, controlled but automated translation process (Huthins, 2010). During its development in 1989s and 1990s, this machine translation began to emerge by using rule-based approaches by which a set of linguistic rules were written manually by linguits and translators for each language pair. Then in the late 1990s, its development got much influence from human translation data which were saved in TM. In the 1990s and 2000s, machine translation got more input from professional human translation in TMs on the area of number of languages, directions, genres, and text types. This was quickly followed by freely available online system such as Google Translate and Microsoft Bing (Doherty, 2016).

Recent systems of this machine contain millions of human-translated sentences from which they learn the probability patterns, while specialized and freely online systems can contain the collated data from thousands of translators over many years. These systems then are continually improving their quality and efficiency to provide more refined and high-quality translation data.

Presently, more provider and web developer have created lots of online translation software which also called as machine translator. These softwares keep improving their quality over time with the purpose to compete their competitors in language translation technologies salles. Moreover, the evaluators for the Translation Software comprovides the following ranking for the top ten commercial translator software products as mention on Hampshire \& Salvia (2010).

Table 1. Commercial Machine Translation Software Ranking

\begin{tabular}{|c|c|}
\hline Rank & Translation Software \\
\hline 1 & Babylon \\
\hline 2 & @prompt \\
\hline 3 & Systran \\
\hline
\end{tabular}




\begin{tabular}{|c|c|}
\hline 4 & Power Translator \\
\hline 5 & Translate Personal \\
\hline 6 & Translution \\
\hline 7 & Lingovsoft \\
\hline 8 & IdiomaX \\
\hline 9 & Word Magic \\
\hline 10 & Nuero Tran \\
\hline
\end{tabular}

This commercial software which cost upward to $\$ 150$, ranked based on the quality of the software and its usability.Due to very high cost, this software only used by limited number of translator. The preference still goes to free machine translation which provide same quality with no cost. The need to find the best free machine translation has lead the translator to function as human evaluators to judge translation by using criteria of applied linguistic ( such as clarity and fidelity) to assess a text on its own benefit.

Hampshire \& Salvia (2010) used HLA methodology which is based on two applied linguistic concepts: clarity and fidelity to make a rank for free machine translation. Clarity which is synonymous with intelligibility, comprehensibility or understandable is defined as the simplicity with which a reader can understand the translation (Fiederer and O'Brien, 2009). Meanwhile fidelity is defined as the condition to which the translated text contain the information as the original.Furthermore, this rank is made by examining some translation result from this software in six different ways: total aggregate, idioms, formal register, lexical ambiguity, phrasal verbs and grammar (word order). As the result, here is the list based on those criterias.

Table 2. Free Machine Translation Software Ranking

\begin{tabular}{|c|c|}
\hline Rank & Free Machine Translator \\
\hline 1 & Google \\
\hline 2 & Babylon \\
\hline 3 & Reverso \\
\hline 4 & Bing \\
\hline 5 & Babelfish* \\
\hline 6 & Systrans \\
\hline 7 & Prompt \\
\hline 8 & Worldlingo \\
\hline 9 & Intertran \\
\hline 10 & Webtrance \\
\hline
\end{tabular}

*Yahoo version

Although google translation is on the top of the rank, it does not mean that another softwares only have weaknesses. All of the application surely have advantages and disadvantages. On Hampshire \& Salvia research result can be seen that some free machine translation are better in translating specific term like idiomatic expression while some others at formal register. Therefore, it is purely the translator preference to choose which software suits their need by considering type of source text to be translated.

\section{Conclusion}

Translating does not only require smart brain of the translator. It includes also the art of the translator which referes to the ability and sensitifity to choose the appropriate method, procedures, tools, dictionary and other assistances to make the translation result becomes acceptable. Moreover, technology is created to ease humans' work and life. The students who take translation study should be aware of which software and application that suit their needs. During their course and training, they should be familiar with all aids that can be used to help their translation works. Utilizing CAT tools and MT can be a smart choice for great assistance from the recent technology development.

\section{References}

Benfoughal, A. (2010). Students' Difficulties and Strategies in Translation. The Case of Third Year Students, Mentoury University Constantine (Doctoral dissertation, Dissertation. University of Constantine).

Campbell, S. (2002). Translation in the context of EFL - the fifth macroskill? TEFLIN, 13(1), 15pp.

Cocci, L. (2009). CAT Tools for Beginner. Translation Journal Volume 13 No. 4. Retrieved from: https://translationjournal.net/journal/50caten.htm

Doherty,S. (2016). The impact of translation technologies on the process and product of translation. International Journal of Communication 10(2016), 947-969. Retrieved from: 
https://www.researchgate.net/publication/284725157 The impact of translation technologies on the process and product of translation

Esselink, B. (2000). A practical guide to localization. Amsterdam, The Netherlands: John Benjamins.

Fernández-Parra, M. (2016). Integrating computer-assisted translation tools into language learning. In A. ParejaLora, C. Calle-Martínez, \& P. Rodríguez-Arancón (Eds), New perspectives on teaching and working with languages in the digital era(pp. 385-396). Dublin: Research-publishing.net. http://dx.doi.org/10.14705/rpnet.2016. tislid2014.450

Hampshire, S., \& Salvia, C. P. (2010). Translation and the Internet: evaluating the quality of free online machine translators. Quaderns: revista de traducció, (17), 197-209.

Kenny, D. (1996). It looks for all the world as if Günter Grass writes in English. Translation Ireland, 10(3), 1213.

O'Reilly, T. (2005). What is Web 2.0: Design patterns and business models for the next generation of software. Retrieved from http://www.oreilly.com/pub/a/web2/archive/what-is-web-20.html

Patience, A. U. (2016). Modern Technology in Translation: Contributions and Limits. World Applied Sciences Journal. IDOSI Publications. DOI:10.5829/idosi.wasj.2016.34.8.15683

Pym, A. (2011). What technology does to translating. The International Journal for Translation \& Interpreting Research. Vol 3, No. 1

$\begin{array}{llllll}\text { Wordfast } & \text { (2013). } & \text { A } & \text { Brosure. } & \text { Retrieved } & \text { from }\end{array}$ https://www.wordfast.com/pdf/FWAP product sheet SCREEN.pdf

Wordfast Classic. (2013). A Brosure. Retrieved from https://www.wordfast.com/pdf/wf brief classic.pdf

Wordfast Pro5. (2017). A Brosure. Retrieved from https://www.wordfast.com/pdf/WF_ProductBrief_Pro5.v5.pdf 